Voix et Images

\title{
V.5 BJ/NBJ : Fiction/théorie, postures ou impostures (?/!)
}

\section{Louise Blouin et Bernard Pozier}

Volume 10, numéro 2, hiver 1985

La barre du jour / La nouvelle barre du jour

URI : https://id.erudit.org/iderudit/013880ar

DOI : https://doi.org/10.7202/013880ar

Aller au sommaire du numéro

\section{Éditeur(s)}

Université du Québec à Montréal

\section{ISSN}

0318-9201 (imprimé)

1705-933X (numérique)

Découvrir la revue

\section{Citer cet article}

Blouin, L. \& Pozier, B. (1985). V.5 BJ/NBJ : Fiction/théorie, postures ou

impostures (?/!). Voix et Images, 10(2), 153-158. https://doi.org/10.7202/013880ar d'utilisation que vous pouvez consulter en ligne.

https://apropos.erudit.org/fr/usagers/politique-dutilisation/ 


\title{
V.5 BJ/NBJ: Fiction/théorie, postures ou impostures (?/!)
}

\author{
par Louise Blouin, Université du Québec à Trois-Rịvières \\ et Bernard Pozier, Collège de Joliette
}

Recevoir un titre-programme pour une rédaction à deux voix: voila une sorte de devoir, ou une sorte de défi, plus ou moins ludique, que ne renierait certes pas l'equipe d'une revue qui provoque un colloque sous une enseigne comme "Vouloir la fiction (c) la modernité».

Ici pour répondre, et comme en plus ce texte accompagnera de savantes études, optons pour la marge, et pour être de bon ton, donnons dans le fragment, allègrement.

\section{.1 - L'imposture du titre}

«LA BARRE DU JOUR», voilà une expression bien québécoise! Cela devrait sans doute annoncer une revue axée sur le folklore, le patrimoine, le pays, la nature... presque tout ce qu'en fait, farouchement, l'on fuit dans la plupart des textes que cela coiffe. Et puis ce fut LA NOUVELLE BARRE DU JOUR, pour marquer un changement d'équipe (heureusement qu'on n'a pas ajouté un adjectif à chaque métamorphose, la couverture déjà serait pleine de "Re-Re-Re nouvelle nouvelle nouvelle BARRE DU JOUR», et l'on sait tous maintenant que le blanc est aussi bavard que le texte parfois, donc sauvons l'espace!). Au moins, ce changement de titre a l'avantage d'éclaircir le sujet (le soleil monte!). "Nouvelle» détermine le champ d'action des manifestations qui s'inscrivent dans les pages. Il s'agirait donc de rénover le langage québécois, ici figuré par l'expression crépusculaire «barre du jour». Et puis il y a cette «barre» dont il faut bien parler et qui bascule sur son horizon pour devenir la barre oblique, symbole en quelque sorte du travail formaliste multipliant les lectures, ouvrant dans les textes les sens les plus divers et les plus inattendus. Et déjà se profile dans le «nouvelle» le «elle» qui déborde dans les années quatre-vingt.

\section{.2 - L'imposture de l'image}

L'aura d'une revue se fabrique à coups de préjugés. On croit toujours que dans telle revue, ça prendra un texte plutôt comme ceci que comme cela, parce qu'on y a lu ou vu des choses dans ce sens, parce que d'autres en ont parlé ainsi ... Finalement, si on veut y publier, on choisira probablement le texte qui ressemblera le plus à cette image qu'on se fait de la revue. On en a même vu écrire de façon à entrer dans tel lieu, à chercher donc à coïncider avec l'image. Pourtant presque toutes les équipes éditoriales, dans presque toutes les revues et dans presque toutes les maisons d'édition, tiennent le 
même discours: «Nous sommes ouverts à toutes les écritures, nous proposons la diversité ... et bla bla bla». Et malgré tout, c'est presque toujours vrai, du moins dans la mesure où l'on peut, la plupart du temps, trouver un certain nombre d'exemples pour contredire toute généralisation théorico-critique du genre: «vous ne publiez que telle sorte de textes». Et pourtant il y a cette magie qui fait que l'on pense toujours tous unanimement et spontanément que tel texte devrait être posté plutôt à la NBJ ou plutôt à Estuaire.

\section{.3 - En posture d'imposture}

Posture BJ/posture NBJ/imposture BJ/imposture NBJ et/ou périple aventureux sur la page-laboratoire où s'exerce la pratique de l'écriture/lecture et/ou lecture/écriture (ouf!). - Ici le comité de rédaction de n'importe quelle équipe BJ/NBJ suggérerait sans doute de biffer cette dernière parenthèse: «guère conforme à l'orientation de notre revue et surprenante par rapport à l'ensemble de votre démarche et ne s'inscrivant pas dans la modernité et tout ça...) Revenons-en au fait, s'il y avait là, dans cet immense travail de produire 140 numéros d'une revue littéraire de fiction au Québec, une certaine posture identifiable, ce serait sârement celle de se placer en état d'écriture, mais comme un ouvrier, une sorte d'entrepreneur en textualité, un fabricant de matériaux textuels. Et s'il y avait imposture, ce serait peut-être face au lecteur, face à celui en tout cas qui n'est pas lui-même un chercheur dans ce domaine, celui qui n'est même pas un amateur dans cette discipline qu'est le «bricolage dans le langage», celui pour qui la langue est encore un outil et pas une matière. Fondamentalement la posture est là, c'est celle du scribe qui grattera des mots dans la pierre, ou encore "mieux», celle du sculpteur qui arrachera à la masse des langages de nouvelles formes, qui y découvrira de nouvelles formules. Et n'oublions pas ce que nous rappelle Yolande Villemaire: «Le scribe est un aveugle qui trace des hiéroglyphes en braille pour arriver à voir ce qui se passe»; lisons à travers les signes!

\section{.4 - Pour une pratique à la mode de}

Si certains parfois peuvent travestir leur écriture pour entrer à la NBJ, d'autres, plus jeunes, y font simplement leurs classes. Comment donc écrire dans la ligne de la NBJ ou dans ce que l'on croit tel, «non loin des coquetteries de Linguistic Parc» comme dirait Michel Gay? Evidemment citer, en commençant, Nicole Brossard par exemple, ne pourrait pas nuire. Mal pris, on pourrait se rabattre sur quelques autres noms québécois habituellement mis en vedette par la revue, mais mieux vaudrait peut-être de gros noms d'intellectuels français ou même américains pourvu que plusieurs les aient déjà nommés dans la revue. Il faut prouver dès l'entrée en matière que l'on a des lettres et les bonnes, sinon comptons sur la vanité et citons carrément un membre du comité de rédaction. Après cela il faut, bien sar, se mettre au texte, mais dans l'économie plutôt que dans la bavardise. Alors nous savons qu'il faut avant tout éviter à tout prix la lisibilité univoque, le 
sens évident. Se ranger plutôt du côté de l'effet apparent. Éviter donc la phrase complète, la ponctuation correcte et la présentation normative de la prose ou du poème. Ce qu'il faut saisir aussi, c'est qu'ici l'intelligence et la réflexion seront toujours privilégiées devant une certaine imagination (au sens d'(images» et d' «imaginaire»), devant aussi une certaine sensibilité, dite de type traditionnel. Il s'agirait en quelque sorte de produire un texte qui soit une sorte de message subliminal, quelque chose où l'idée que l'on se fait de son propre texte et la pensée de ce qu'on voudrait y avoir fait sont définitivement plus importantes que ce que l'on met réellement sur la page, en tout cas que ce que le lecteur peut y déceler. L'écriture y serait donc, d'une certaine façon, le signe qui vient voiler l'intention ou les motifs de l'inscription. Est-ce là une posture? Serait-ce une imposture? Et pour qui? Continuons tout de même à jouer du cliché et du préjugé pour esquisser une caricature-robot du disciple de la NBJ: voyons où cela nous mène? (Doit-on d'ailleurs aboutir?) Ce qu'on retient aussi de l'image diffusée autour de la NBJ, ce serait les étiquettes identifiant quelques courants comme LE formalisme, LE féminisme, LE modernisme... avec chacun leurs thèmes, leurs mots, leur quincaillerie, leurs canons, leurs habitudes. Puisons-y généreusement! Qu'utiliser aussi pour faire NBJ? (Attention! toutes ces recettes sont vraies et en même temps completement fausses!) Assaisonnons avec une sorte du ludisme, mais à froid; pratiquons un jeu sérieux, sans humour trop direct, sans véritable fantaisie (au sens traditionnel, il va sans dire!). Il faudra pouvoir rire chez soi, dans sa tête, à propos de son idée brillante qui a déjoué tout le monde, qui a trompé tous les lecteurs, à tel point 'qu'on est le seul à le savoir! (peut-être!) Ensuite il faudrait, bien sûr, appliquer LE modele, puisque c'est là l'apport primordial de la NBJ. Elle a favorisé la création d'un nouveau genre littéraire, le TEXTE, sorte de bloc ou de fragment de théorie/fiction-prose/poème. Même si cela mélange bien des chroniqueurs, quelques professeurs, certains éditeurs et plusieurs jurys, il faut convenir que, comme le souligne avec raison Jean Yves Collette (NBJ, no 141), il y a là émergence d'une particularité très spécifique de l'écriture québécoise contemporne. Et un peu plus de conscience de ce que l'on a réussi pourrait en faire ici un certain pendant de ce qui est arrivé aux Etats-Unis pour la SHORT STORY. Voilà une posture à prendre! Concrète et «moderne»!

\section{.5 - La fiction comme posture}

140 numéros de revue, si l'on suppose en moyenne 100 pages par numéro, cela donne aux alentours de 14,000 pages de textes: une somme fort respectable que plusieurs petits éditeurs de poésie n'atteignent sans doute pas dans le même laps de temps. Il y a donc là un immense apport à notre littérature, 'autant par les possibilités offertes aux auteurs éventuels d'y publier quelques textes que par l'éventail et la diversité des noms et des écritures ainsi rendus publics. Il faut souligner aussi, ce qui n'est pas fait souvent, l'infini travail que cela suppose au hiveau éditorial: animation, sélection, discussion, correction, mise en page, stimulation, etc. C'est un des hommages qu'il nous faut rendre à tous ces écrivains qui ont aujourd'hui à peu près entre 35 et 45 ans, ils sont en général très intelligents et en plus ils ont beaucoup travaillé, non seulement pour écrire mais pour 
fabriquer une véritable vie littéraire avec des revues, des maisons d'édition, des colloques, des lieux critiques, etc. Nous pensons ici par exemple aux Nicole Brossard, France Théoret, Jean Yves Collette, Michel Gay, Claude Beausoleil, André Roy, Normand de Bellefeuille, Michel Beaulieu... Il faut certes aussi célébrer l'esprit de nouveauté qui a animé les différentes équipes et ce, même si les manifestations qui en découlent peuvent être discutées dans tous leurs excès. Il y a là un esprit et un enthousiasme immenses pour la chose fictionnelle, et ces attitudes-là, dans l'histoire de notre littérature, sont désormais indéniables, inébranlables, ineffaçables.

\section{.6 - Dissémination de la théorie}

Qu'on l'appelle fiction théorique ou théorie-fiction, le TEXTE, comme genre litteraire, s'affiche de toute façon comme une production hybride: qu'on songe par exemple à tous ces problèmes de classification que suscitent des ouvrages comme Picture. Theory, la Parole verte ou le Livre du devoir; qu'on songe aussi à la vague de réflexion sur le travail d'écriture qui a caractérisé les textes poétiques et fictionnels des années soixante-dix. En effet depuis environ une quinzaine d'années, la théorie se cache et se révèle dans les pratiques des écritures de fiction; à peu près jamais, elle ne se retrouve dans des essais, et si cela peut être un avantage pour l'intérêt du lecteur/écrivant, cela contrarie évidemment tous ceux qui veulent comprendre et qui ont à jouer aux chercheurs d'or pour démêler dans les masses textuelles quelques pépites éclairantes. Comment trouver des ouvrages de références qui nous expliqueraient clairement la modernité québécoise et ses pratiques? Il faut avouer que les manuels sont rares et qu'il faut plutôt chercher des pistes de réponses à travers les textes mêmes des poètes et/ou romanciers. Alors on cite Blanchot, Deleuze, Lyotard, Guattari, Barthes, Cixous; Kristeva, Wittgenstein, Cooper, Lupasco ... ou bien un vers de Beausoleil, quelques lignes de Nicole Brossard ou un autre éclat allusif ou métaphorique tiré d'un fragment fictionnel, peut-être un flash coloré de Yolande Villemaire, ou une sentence bien frappée de Paul Chamberland! Peut-être serait-il temps de ramasser un peu toutes nos découvertes de ces folles et passionnantes années de la littérature québécoise: il y en a plusieurs, et des phénoménales! Pourquoi ne pas dire maintenant à tous, et lisiblement, ce que certains ont imaginé? Et comme le disait Miron: «quand elle sera connue, la littérature québécoise étonnera le monde». Et nous aurons l'immense avantage que ces théories-là seront nées de la pratique et $y$ trouveront leur fondements et leurs preuves, nous n'aurons même pas besoin de faire comme les essayistes français qui nous racontent, a peu près tous, les deux geurres mondiales, la révolution française et mai soixante-huit, avant de dire quoi que ce soit; nous, nous avons tant à dire tellement il s'est imaginé de choses dans les pages de nos textes! Voilà une deuxième posture à prendre, et qui impose le genre!

\section{. 7 - L'imposture institutionnelle}

La BJ et la NBJ ont joué le pari du travail formel et parfois le jeu de l'illisible et, au fond, elles ont gagné, parce que, paradoxalement, cette 
avant-garde a joué le jeu institutionnel. Aujourd'hui la NBJ est certes une des revues les plus subventionnées, elle a une grosse distribution chez les écrivains mais aussi dans les bibliothèques et dans les organismes culturels, et c'est un produit très «clean». Ses dirigeants aussi prennent parfois l'aspec et le comportement apparent de véritables petits fonctionnaires de la culture (rien à voir avec l'effet $H o b o-Q u e ́ b e c, C u l Q$; aucune ressemblance avec l'attitude Jean Leduc). Bien sur, il ne faut pas leur en vouloir, il faudrait même souligner leur brio à ce jeu, puisqu'ils permettent ainsi que survivent très bien dans l'Institution des pratiques para-institutionnelles. Mais il faut cependant être bien conscient de ce paradoxe et de ses conséquences: par exemple, d'être devenu une sorte de classicisme d'une certaine avant-garde et de rejeter tout ce qui en dépasse par des charges ou par des outrances. Les textes de la NBJ sont «modernes», ils délirent, mais ils n'attaquent rien, ne ridiculisent pas, ne provoquent pas et, finalement, ne dérangent pas cette institution qu'au niveau du fonctionnement on plagie en imposture. Cette avant-garde-là est toujours subventionnable et toujours assimable et ça n'ennuie aucun fonctionnaire que la revue soit riche et partout, puisque justement pour ces gens-là, dans les textes de la NBJ, on ne «dit» rien, il n'y a rien à "comprendre». (En jouant à la bureaucratie, on en vient même à être capable de jouer a l'auto-censure: par exemple à refuser des textes ou des numéros spéciaux que l'on a commandés, ou même à camoufler les événements que l'on crée. Ainsi on lit sur le numéro 90-91 «colloque nbj 1980 »; sur le numéro 118-119 «colloque nbj 1982», et puis sur le numéro 141 «deuxième colloque bissiextile». Bien sûr, ce n'est pas vraiment faux; bien sar, on joue sur les mots, mais cela ressemble finalement à de la dissimulation puisqu'on retient davantage le "deuxième» que le «bissextile». On dira que c'était pour rire, mais cela ressemble tout de même aux pratiques d'un comptable qui veut sauver de l'impôt! Que veulent donc sauver les membres du comité de censure de la NBJ en tentant de nous faire oublier le colloque consacré à Nicole Brossard qui avait été organisé pour la revue par Claude Beausoleil? (Pour une cause ou par humour, en posture ou en imposture, dans ces bureaux-là; en tout cas, on joue la carte du pouvoir!)

\section{.8 - La Barre du Jour}

La BJ est partie de «La Littérature Québécoise» qui était faite, accessible, peu axée sur les recherches et les expérimentations, mais plutôt sur l'histoire et le présent, dans une pratique de témoignage. Elle a donc un peu participé au début à une certaine pratique qui proposait, en littérature comme ailleurs, d'abord et avant tout d'être. On y a donc aperçu des textes et des auteurs dits traditionnels et même engagés. Mais on y a vite choisi aussi, et surtout, d'être des détecteurs d'impostures, celles justement de la tradition; et d'amener des nouveaux imposteurs, ouvrant dans d'autres directions, proposant l'exploration du langage. Ceux-ci alors devinrent des stimuli pour mettre en posture de nouvelles lectures / écritures, pour forger de nouvelles impostures. Il s'agissait donc d'abord de débroussailler, d'ouvrir à l'écriture d'ici de nouveaux champs, de sortir du noir pour 
brandir nos étincelles, de «bruts bruits de neuf», comme disait Gatien Lapointe.

\section{9 - La Nouvelle Barre du Jour}

De par le programme même de son titre, la NBJ a voulu reprendre à son compte une démarche déjà esquissée par. la BJ, plutôt que de fonder du neuf. En ce sens d'ailleurs, elle repose sur une sorte de tradition du moderne. Alors elle n'a plus de souci historique, ni de bris a brandir, puisqu'elle vient s'inscrire dans une littérature québécoise qui existe déjà et dans un sillon déjà tracé. Elle est donc menacée dans son propos même d'un certain et relatif statu quo, c'est-à-dire d'être là finalement pour encourager la reproduction et le développement d'une certaine veine textuelle déjà amorcée, et son défi est de la pousser vers des ailleurs multiples et des devenirs pluriels. Mais dans cette entreprise, ce qui la menace, c'est la forme de l'entonnoir et le risque de sombrer dans un certain gargarisme de son académisme déjà présent (et c'est contre cela que doivent toujours lutter les avant-gardes). Il faut sans cesse se défier du fait que parfois la mode puisse prendre le pas sur la modernité tant recherchée. Et comme on date très vite, surtout en cette fin de siècle, il faut rester alerte car bien vite la peur du risque et celle de la montée des différences peuvent biaiser les regards. Déjà les textes des moins de 35 ans diffèrent de ceux de leurs aînés, à moins qu'ils n'aient été des élèves un peu trop appliqués. "Chaque génération se doit de récuser la précédente si elle ne tient pas à l'imiter", nous rappelle avec sagesse monsieur Clément Marchand. Une posture à prendre, ce serait donc aussi celle de la diversité véritable, le TEXTE se devrait d'être aussi cosmopolite que la ville qu'il célèbre si souvent, mais ce n'est pas toujours le cas parce qu'on choisit parfois un peu trop en fonction de soi-même, avec ses critères de «qualité» qui risquent de ressembler souvent à nos propres gouts, à nos propres pratiques. Il faut aussi publier nos contraires et même nos adversaires, pour une véritable polysémie des textures plurielles s'éclatant vers toutes les fictions du possible.

\section{.10 - Et le nouveau lisible}

On dit qu'il y a de nos jours un retour au lyrisme, au romantisme et a la lisibilité: là aussi, c'est vrai et c'est faux. Et à la NBJ comme ailleurs, la traversée du langage n'est pas encore totalement faite. Et heureusement on n'est pas près d'y dire que cette supposée «lisibilité», ce serait la facilité, car sans véritable recherche il n'y a pas d'écriture même s'il peut y avoir du discours, de la parole. Alors les mots à saisir au bout du tunnel formel sont ceux d'une lumière noire qui jette dans nos yeux les éclats intenses des tragédies de notre siècle et de ses petites splendeurs. Car «maintenant les formes parlent et c'est dans cette direction que les surprises abonderont», prédit Claude Beausoleil. Finalement l'apport immense de la BJ/NBJ aura été de nous faire prendre conscience de ces formes-là avec lesquelles on peut dire, de nous faire évaluer les jeux et les enjeux de ces quelques paquets de signes avec lesquels refaire le monde sur les pages, si on ne peut le faire ailleurs, et enfin de remettre en nous les mots pour que l'on puisse jouer sa vie et son éternité. 